\title{
AVERAGE NON-VANISHING OF DIRICHLET L-FUNCTIONS AT THE CENTRAL POINT
}

\author{
KYLE PRATT
}

\begin{abstract}
The Generalized Riemann Hypothesis implies that at least $50 \%$ of the central values $L\left(\frac{1}{2}, \chi\right)$ are non-vanishing as $\chi$ ranges over primitive characters modulo $q$. We show that one may unconditionally go beyond GRH, in the sense that if one averages over primitive characters modulo $q$ and averages $q$ over an interval, then at least $50.073 \%$ of the central values are non-vanishing. The proof utilizes the mollification method with a three-piece mollifier, and relies on estimates for sums of Kloosterman sums due to Deshouillers and Iwaniec.
\end{abstract}

\section{InTRODUCTION}

It is widely believed that no primitive Dirichlet $L$-function $L(s, \chi)$ vanishes at the central point $s=\frac{1}{2}$. Most of the progress towards this conjecture has been made by working with various families of Dirichlet $L$-functions. Balasubramanian and Murty [1] showed that, in the family of primitive characters modulo $q$, a positive proportion of the $L$-functions do not vanish at the central point. Iwaniec and Sarnak [10] later improved this lower bound, showing that at least $\frac{1}{3}$ of the $L$-functions in this family do not vanish at the central point. Bui [4] improved this further to $34.11 \%$, and Khan and Ngô [11] showed at least $\frac{3}{8}$ of the central values are non-vanishing 1 for prime moduli. Soundararajan [16] worked with a family of quadratic Dirichlet characters, and showed that $\frac{7}{8}$ of the family do not vanish at $s=\frac{1}{2}$. These proofs all proceed through the mollification method, which we discuss in section 2 below.

If one assumes the Generalized Riemann Hypothesis, one can show that at least half of the primitive characters $\chi(\bmod q)$ satisfy $L\left(\frac{1}{2}, \chi\right) \neq 0$ [1, 14], [13, Exercise 18.2.8]. One uses the explicit formula, rather than mollification, and the proportion $\frac{1}{2}$ arises from the choice of a test function with certain positivity properties.

It seems plausible that one may obtain a larger proportion of non-vanishing by also averaging over moduli $q$. Indeed, Iwaniec and Sarnak [10] already claimed that by averaging over moduli one can prove at least half of the central values are nonzero. This is striking, in that it is as strong, on average, as the proportion obtained via GRH.

A natural question is whether, by averaging over moduli, one can breach the $50 \%$ barrier, thereby going beyond the immediate reach of GRH. We answer this question in the affirmative.

Let $\sum_{\chi(q)}^{*}$ denote a sum over the primitive characters modulo $q$, and define $\varphi^{*}(q)$ to be the number of primitive characters modulo $q$.

2010 Mathematics Subject Classification. 11M06, 11M26.

Keywords and phrases: Dirichlet $L$-function, non-vanishing, central point, mollifier, sums of Kloosterman sums.

${ }^{1}$ A clear preference for "non-vanishing" or "nonvanishing" has not yet materialized in the literature. We exclusively use the former term throughout this work. 
Theorem 1.1. Let $\Psi$ be a fixed, nonnegative smooth function, compactly supported in $\left[\frac{1}{2}, 2\right]$, which satisfies

$$
\int_{\mathbb{R}} \Psi(x) d x>0 .
$$

Then for $Q$ sufficiently large we have

$$
\sum_{q} \Psi\left(\frac{q}{Q}\right) \frac{q}{\varphi(q)} \sum_{\substack{\chi(q) \\ L\left(\frac{1}{2}, \chi\right) \neq 0}}^{*} 1 \geq 0.50073 \sum_{q} \Psi\left(\frac{q}{Q}\right) \frac{q}{\varphi(q)} \varphi^{*}(q) .
$$

Thus, roughly speaking, a randomly chosen central value $L\left(\frac{1}{2}, \chi\right)$ is more likely nonzero than zero. We remark also that the appearance of the arithmetic weight $\frac{q}{\varphi(q)}$ is technically convenient, but not essential.

\section{Mollification, And A SKetch For Theorem 1.1}

The proof of Theorem 1.1 relies on the powerful technique of mollification. For each character $\chi$ we associate a function $\psi(\chi)$, called a mollifier, that serves to dampen the large values of $L\left(\frac{1}{2}, \chi\right)$. By the Cauchy-Schwarz inequality we have

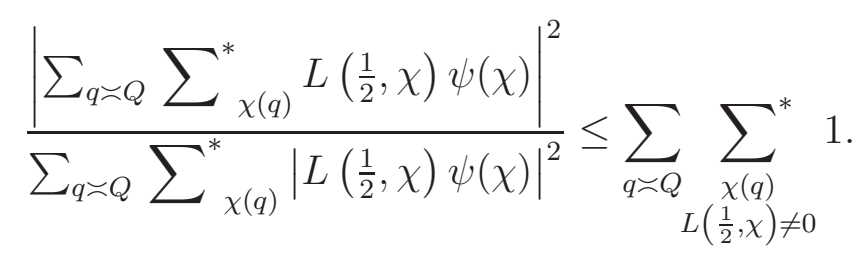

The better the mollification by $\psi$, the larger proportion of non-vanishing one can deduce.

It is natural to choose $\psi(\chi)$ such that

$$
\psi(\chi) \approx \frac{1}{L\left(\frac{1}{2}, \chi\right)} .
$$

Since $L\left(\frac{1}{2}, \chi\right)$ can be written as a Dirichlet series

$$
L\left(\frac{1}{2}, \chi\right)=\sum_{n=1}^{\infty} \frac{\chi(n)}{n^{\frac{1}{2}}}
$$

this suggests the choice

$$
\psi(\chi) \approx \sum_{\ell \leq y} \frac{\mu(\ell) \chi(\ell)}{\ell^{\frac{1}{2}}} .
$$

We have introduced a truncation $y$ in anticipation of the need to control various error terms that will arise. We write $y=Q^{\theta}$, where $\theta>0$ is a real number. At least heuristically, larger values of $\theta$ yield better mollification by (2.3). Iwaniec and Sarnak [10] made this choice (2.3) (up to some smoothing), and found that the proportion of non-vanishing attained was

$$
\frac{\theta}{1+\theta} \text {. }
$$

When $\theta=1$ we see (2.4) is exactly $\frac{1}{2}$, so we need $\theta>1$ in order to conclude Theorem 1.1. This seems beyond the range of present technology. Without averaging over moduli we may 
take $\theta=\frac{1}{2}-\varepsilon$, and the asymptotic large sieve of Conrey, Iwaniec, and Soundararajan [6] allows one to take $\theta=1-\varepsilon$ if one averages over moduli. This just falls short of our goal.

Thus, a better mollifier than (2.3) is required. Part of the problem is that (2.2) is an inefficient representation of $L\left(\frac{1}{2}, \chi\right)$. A better representation of $L\left(\frac{1}{2}, \chi\right)$ may be obtained through the approximate functional equation, which states

$$
L\left(\frac{1}{2}, \chi\right) \approx \sum_{n \leq q^{1 / 2}} \frac{\chi(n)}{n^{\frac{1}{2}}}+\epsilon(\chi) \sum_{n \leq q^{1 / 2}} \frac{\bar{\chi}(n)}{n^{\frac{1}{2}}} .
$$

Here $\epsilon(\chi)$ is the root number, which is a complex number of modulus 1 defined by

$$
\epsilon(\chi)=\frac{1}{q^{\frac{1}{2}}} \sum_{h(\bmod q)} \chi(h) e\left(\frac{h}{q}\right) .
$$

Inspired by (2.5), Michel and VanderKam [12] chose a mollifier

$$
\psi(\chi) \approx \sum_{\ell \leq y} \frac{\mu(\ell) \chi(\ell)}{\ell^{\frac{1}{2}}}+\bar{\epsilon}(\chi) \sum_{\ell \leq y} \frac{\mu(\ell) \bar{\chi}(\ell)}{\ell^{\frac{1}{2}}} .
$$

We note that Soundararajan [15] earlier used a mollifier of this shape in the context of the Riemann zeta function.

For $y=Q^{\theta}$, Michel and VanderKam found that (2.7) gives a non-vanishing proportion of

$$
\frac{2 \theta}{1+2 \theta} \text {. }
$$

Thus, we need $\theta=\frac{1}{2}+\varepsilon$ in order for (2.8) to imply a proportion of non-vanishing greater than $\frac{1}{2}$. However, the more complicated nature of the mollifier (2.7) means that, without averaging over moduli, only the choice $\theta=\frac{3}{10}-\varepsilon$ is acceptable [11].

As we allow ourselves to average over moduli, however, one might hope to obtain (2.8) for $\theta=\frac{1}{2}+\varepsilon$. Again we fall just short of our goal. Using a powerful result of Deshouillers and Iwaniec on cancellation in sums of Kloosterman sums (see Lemma 5.1 below) we shall show that $\theta=\frac{1}{2}-\varepsilon$ is acceptable, but increasing $\theta$ any further seems very difficult. It follows that we need any extra amount of mollification in order to obtain a proportion of non-vanishing strictly greater than $\frac{1}{2}$.

The solution is to attach yet another piece to the mollifier $\psi(\chi)$, but here we wish for the mollifier to have a very different shape from (2.7). Such a mollifier was utilized by Bui [4], who showed that

$$
\psi_{\mathrm{B}}(\chi) \approx \frac{1}{\log y} \sum_{b c \leq y} \sum_{\frac{\Lambda(b) \mu(c) \bar{\chi}(b) \chi(c)}{(b c)^{\frac{1}{2}}}}
$$

is a mollifier for $L\left(\frac{1}{2}, \chi\right)$. It turns out that adding (2.9) to (2.7) gives a sufficient mollifier to conclude Theorem 1.1 ,

We remark that, in the course of the proof, the main terms are easily extracted and we have no need here for the averaging over moduli. We require the averaging over moduli in order to estimate some of the error terms.

The structure of the remainder of the paper is as follows. In section 3 we reduce the proof of Theorem 1.1 to two technical results, Lemma 3.3 and Lemma 3.4, which give asymptotic evaluations of certain mollified sums. In section 4 we extract the main term of Lemma 3.3. and in section 5 we use estimates on sums of Kloosterman sums to complete the proof of 
this lemma. Section 6 similarly proves the main term of Lemma 3.4, but this derivation is longer than that given in section 4 because the main terms are more complicated. In the final section, section 7, we bound the error term in Lemma 3.4, again using results on sums of Kloosterman sums.

\section{Proof of Theorem 1.1: First Steps}

Let us fix some notation and conventions that shall hold for the remainder of the paper.

The notation $a \equiv b(q)$ means $a \equiv b(\bmod q)$, and when $a(q)$ occurs beneath a sum it indicates a summation over residue classes modulo $q$.

We denote by $\epsilon$ an arbitrarily small positive quantity that may vary from one line to the next, or even within the same line. Thus, we may write $X^{2 \epsilon} \leq X^{\epsilon}$ with no reservations.

We need to treat separately the even primitive characters and odd primitive characters. We focus exclusively on the even primitive characters, since the case of odd characters is nearly identical. We write $\sum_{\chi(q)}^{+}$for a sum over even primitive characters modulo $q$, and we write $\varphi^{+}(q)$ for the number of such characters. Observe that $\varphi^{+}(q)=\frac{1}{2} \varphi^{*}(q)+O(1)$.

We shall encounter the Ramanujan sum $c_{q}(n)$ (see the proof of Proposition 5.2), defined by

$$
c_{q}(n)=\sum_{\substack{a(q) \\(a, q)=1}} e\left(\frac{a n}{q}\right) .
$$

We shall only need to know that $c_{q}(1)=\mu(q)$ and $\left|c_{q}(n)\right| \leq(q, n)$, where $(q, n)$ is the greatest common divisor of $q$ and $n$.

We now fix a smooth function $\Psi$ as in the statement of Theorem 1.1, and allow all implied constants to depend on $\Psi$. We let $Q$ be a large real number, and set $y_{i}=Q^{\theta_{i}}$ for $i \in\{1,2,3\}$, where $0<\theta_{i}<\frac{1}{2}$ are fixed real numbers. We further define $L=\log Q$. The notation $o(1)$ denotes a quantity that goes to zero as $Q$ goes to infinity.

Let us now begin the proof of Theorem 1.1 in earnest. As discussed in section 2, we choose our mollifier $\psi(\chi)$ to have the form

$$
\psi(\chi)=\psi_{\mathrm{IS}}(\chi)+\psi_{\mathrm{B}}(\chi)+\psi_{\mathrm{MV}}(\chi),
$$

where

$$
\begin{aligned}
\psi_{\mathrm{IS}}(\chi) & =\sum_{\ell \leq y_{1}} \frac{\mu(\ell)}{\ell^{\frac{1}{2}}} P_{1}\left(\frac{\log \left(y_{1} / \ell\right)}{\log y_{1}}\right) \\
\psi_{\mathrm{B}}(\chi) & =\frac{1}{L} \sum_{b c \leq y_{2}} \sum_{\Lambda(b) \mu(c) \bar{\chi}(b) \chi(c)} P_{2}\left(\frac{\log \left(y_{2} / b c\right)}{\log y_{2}}\right) \\
\psi_{\mathrm{MV}}(\chi) & =\epsilon(\bar{\chi}) \sum_{\ell \leq y_{3}} \frac{\mu(\ell) \bar{\chi}(\ell)}{\ell^{\frac{1}{2}}} P_{3}\left(\frac{\log \left(y_{3} / \ell\right)}{\log y_{3}}\right)
\end{aligned}
$$

The smoothing polynomials $P_{i}$ are real and satisfy $P_{i}(0)=0$. For notational convenience we write

$$
P_{i}\left(\frac{\log \left(y_{i} / x\right)}{\log y_{i}}\right)=P_{i}[x]
$$


There is some ambiguity in this notation because of the $y_{i}$-dependence in the polynomials, and this needs to be remembered in calculations.

Now define sums $S_{1}$ and $S_{2}$ by

$$
\begin{aligned}
& S_{1}=\sum_{q} \Psi\left(\frac{q}{Q}\right) \frac{q}{\varphi(q)} \sum_{\chi(q)}^{+} L\left(\frac{1}{2}, \chi\right) \psi(\chi), \\
& S_{2}=\sum_{q} \Psi\left(\frac{q}{Q}\right) \frac{q}{\varphi(q)} \sum_{\chi(q)}^{+}\left|L\left(\frac{1}{2}, \chi\right) \psi(\chi)\right|^{2} .
\end{aligned}
$$

We apply Cauchy-Schwarz as in (2.1) and get

$$
\sum_{q} \Psi\left(\frac{q}{Q}\right) \frac{q}{\varphi(q)} \sum_{\substack{\chi(q) \\ L\left(\frac{1}{2}, \chi\right) \neq 0}}^{+} 1 \geq \frac{S_{1}^{2}}{S_{2}}
$$

The proof of Theorem 1.1 therefore reduces to estimating $S_{1}$ and $S_{2}$. We obtain asymptotic formulas for these two sums.

Lemma 3.1. Suppose $0<\theta_{1}, \theta_{2}<1$ and $0<\theta_{3}<\frac{1}{2}$. Then

$$
S_{1}=\left(P_{1}(1)+P_{3}(1)+\frac{\theta_{2}}{2} \widetilde{P}_{2}(1)+o(1)\right) \sum_{q} \Psi\left(\frac{q}{Q}\right) \frac{q}{\varphi(q)} \varphi^{+}(q),
$$

where

$$
\widetilde{P_{2}}(x)=\int_{0}^{x} P_{2}(u) d u .
$$

Lemma 3.2. Let $0<\theta_{1}, \theta_{2}, \theta_{3}<\frac{1}{2}$ with $\theta_{2}<\theta_{1}, \theta_{3}$. Then

$$
S_{2}=\left(2 P_{1}(1) P_{3}(1)+P_{3}(1)^{2}+\frac{1}{\theta_{3}} \int_{0}^{1} P_{3}^{\prime}(x)^{2} d x+\kappa+\lambda+o(1)\right) \sum_{q} \Psi\left(\frac{q}{Q}\right) \frac{q}{\varphi(q)} \varphi^{+}(q),
$$

where

$$
\kappa=3 \theta_{2} P_{3}(1) \widetilde{P_{2}}(1)-2 \theta_{2} \int_{0}^{1} P_{2}(x) P_{3}(x) d x
$$

and

$$
\begin{aligned}
\lambda & =P_{1}(1)^{2}+\frac{1}{\theta_{1}} \int_{0}^{1} P_{1}^{\prime}(x)^{2} d x-\theta_{2} P_{1}(1) \widetilde{P_{2}}(1)+2 \theta_{2} \int_{0}^{1} P_{1}\left(1-\frac{\theta_{2}(1-x)}{\theta_{1}}\right) P_{2}(x) d x \\
& +\frac{\theta_{2}}{\theta_{1}} \int_{0}^{1} P_{1}^{\prime}\left(1-\frac{\theta_{2}(1-x)}{\theta_{1}}\right) P_{2}(x) d x+\theta_{2}^{2} \int_{0}^{1}(1-x) P_{2}(x)^{2} d x \\
& +\frac{\theta_{2}}{2} \int_{0}^{1}(1-x)^{2} P_{2}^{\prime}(x)^{2} d x-\frac{\theta_{2}^{2}}{4} \widetilde{P_{2}}(1)^{2}+\frac{\theta_{2}}{4} \int_{0}^{1} P_{2}(x)^{2} d x .
\end{aligned}
$$

Proof of Theorem 1.1. Lemmas 3.1 and 3.2 give the evaluations of $S_{1}$ and $S_{2}$ for even characters. The identical formulas hold for odd characters. Theorem 1.1 then follows from (3.4) 
upon choosing $\theta_{1}=\theta_{3}=\frac{1}{2}, \theta_{2}=0.163$, and

$$
\begin{aligned}
& P_{1}(x)=4.86 x+0.29 x^{2}-0.96 x^{3}+0.974 x^{4}-0.17 x^{5}, \\
& P_{2}(x)=-3.11 x-0.3 x^{2}+0.87 x^{3}-0.18 x^{4}-0.53 x^{5} \\
& P_{3}(x)=4.86 x+0.06 x^{2}
\end{aligned}
$$

These choices actually yield a proportion?

$$
\geq 0.50073004 \ldots,
$$

which allows us to state Theorem 1.1 with a clean inequality.

We note without further comment the curiosity in the proof of Theorem 1.1 that the largest permissible value of $\theta_{2}$ is not optimal.

We can dispense with $S_{1}$ quickly.

Proof of Lemma 3.1. Apply [4, Theorem 2.1] and the argument of [12, section 3], using the facts $L=\log q+O(1)$ and $y_{i}=q^{\theta_{i}+o(1)}$.

The analysis of $S_{2}$ is much more involved, and we devote the remainder of the paper to this task. We first observe that (3.1) yields

$$
|\psi(\chi)|^{2}=\left|\psi_{\mathrm{IS}}(\chi)+\psi_{\mathrm{B}}(\chi)\right|^{2}+2 \operatorname{Re}\left\{\psi_{\mathrm{IS}}(\chi) \psi_{\mathrm{MV}}(\bar{\chi})+\psi_{\mathrm{B}}(\chi) \psi_{\mathrm{MV}}(\bar{\chi})\right\}+\left|\psi_{\mathrm{MV}}(\chi)\right|^{2} .
$$

By [4, Theorem 2.2] we have

$$
\sum_{\chi(q)}^{+}\left|L\left(\frac{1}{2}, \chi\right)\right|^{2}\left|\psi_{\mathrm{IS}}(\chi)+\psi_{\mathrm{B}}(\chi)\right|^{2}=\lambda \varphi^{+}(q)+O\left(q L^{-1+\epsilon}\right),
$$

where $\lambda$ is as in Lemma 3.2. We also have

$$
\begin{aligned}
\frac{1}{\varphi^{+}(q)} \sum_{\chi(q)}^{+}\left|L\left(\frac{1}{2}, \chi\right)\right|^{2}\left|\psi_{\mathrm{MV}}(\chi)\right|^{2} & =\frac{1}{\varphi^{+}(q)} \sum_{\chi(q)}^{+}\left|L\left(\frac{1}{2}, \chi\right)\right|^{2}\left|\sum_{\ell \leq y_{3}} \frac{\mu(\ell) \chi(\ell) P_{3}[\ell]}{\ell^{\frac{1}{2}}}\right|^{2} \\
& =P_{3}(1)^{2}+\frac{1}{\theta_{3}} \int_{0}^{1} P_{3}^{\prime}(x)^{2} d x+O\left(L^{-1+\epsilon}\right),
\end{aligned}
$$

by the analysis of the Iwaniec-Sarnak mollifier (see [4, section 2.3]).

Therefore, in order to prove Lemma 3.2 it suffices to prove the following two results.

Lemma 3.3. For $0<\theta_{1}, \theta_{3}<\frac{1}{2}$ we have

$$
\begin{aligned}
\sum_{q} \Psi\left(\frac{q}{Q}\right) \frac{q}{\varphi(q)} \sum_{\chi(q)}^{+}\left|L\left(\frac{1}{2}, \chi\right)\right|^{2} \psi_{I S}(\chi) \psi_{M V}(\bar{\chi}) \\
=\left(P_{1}(1) P_{3}(1)+o(1)\right) \sum_{q} \Psi\left(\frac{q}{Q}\right) \frac{q}{\varphi(q)} \varphi^{+}(q) .
\end{aligned}
$$

\footnotetext{
${ }^{2} \mathrm{~A}$ Mathematica $\mathrm{R}$, file with this computation is included with this paper on arxiv.org.
} 
Lemma 3.4. Let $0<\theta_{2}<\theta_{3}<\frac{1}{2}$. Then

$$
\begin{aligned}
\sum_{q} \Psi & \left(\frac{q}{Q}\right) \frac{q}{\varphi(q)} \sum_{\chi(q)}^{+}\left|L\left(\frac{1}{2}, \chi\right)\right|^{2} \psi_{B}(\chi) \psi_{M V}(\bar{\chi}) \\
& =\left(\frac{3 \theta_{2}}{2} P_{3}(1) \widetilde{P_{2}}(1)-\theta_{2} \int_{0}^{1} P_{2}(x) P_{3}(x) d x+o(1)\right) \sum_{q} \Psi\left(\frac{q}{Q}\right) \frac{q}{\varphi(q)} \varphi^{+}(q) .
\end{aligned}
$$

\section{LEMMA 3.3 MAIN TERM}

The goal of this section is to extract the main term in Lemma 3.3. The main term analysis is given in [12, section 6], but as the ideas also appear in the proof of Lemma 3.4 we give details here.

We begin with two lemmas.

Lemma 4.1. Let $\chi$ be a primitive even character modulo q. Let $G(s)$ be an even polynomial satisfying $G(0)=1$, and which vanishes to second order at $\frac{1}{2}$. Then we have

$$
\left|L\left(\frac{1}{2}, \chi\right)\right|^{2}=2 \sum_{m, n} \sum_{\frac{\chi}{(m)} \bar{\chi}(n)} V\left(\frac{m n}{q}\right),
$$

where

$$
V(x)=\frac{1}{2 \pi i} \int_{(1)} \frac{\Gamma^{2}\left(\frac{s}{2}+\frac{1}{4}\right)}{\Gamma^{2}\left(\frac{1}{4}\right)} \frac{G(s)}{s} \pi^{-s} x^{-s} d s .
$$

Proof. See [10, (2.5)]. The result follows along the lines of [9, Theorem 5.3].

We remark that $V$ satisfies $V(x) \ll_{A}(1+x)^{-A}$, as can be seen by moving the contour of integration to the right. We also note that the choice of $G(s)$ in Lemma 4.1 is almost completely free. In particular, we may choose $G$ to vanish at whichever finite set of points is convenient for us (see (4.6) below for an application).

Lemma 4.2. Let $(m n, q)=1$. Then

$$
\sum_{\chi(q)}^{+} \chi(m) \bar{\chi}(n)=\frac{1}{2} \sum_{\substack{v w=q \\ w \mid m \pm n}} \sum_{w} \mu(v) \varphi(w) .
$$

Proof. See [5, Lemma 4.1], for instance.

We do not need the averaging over $q$ in order to extract the main term of Lemma 3.3. We insert the definitions of the mollifiers $\psi_{\mathrm{IS}}(\chi)$ and $\psi_{\mathrm{MV}}(\bar{\chi})$, then apply Lemma 4.1 and interchange orders of summation. We obtain

$$
\begin{aligned}
& \sum_{\chi(q)}^{+}\left|L\left(\frac{1}{2}, \chi\right)\right|^{2} \psi_{\mathrm{IS}}(\chi) \psi_{\mathrm{MV}}(\bar{\chi}) \\
& =2 \sum_{\substack{\ell_{1} \leq y_{1} \\
\ell_{3} \leq y_{3} \\
\left(\ell_{1} \ell_{3}, q\right)=1}} \frac{\mu\left(\ell_{1}\right) \mu\left(\ell_{3}\right) P_{1}\left[\ell_{1}\right] P_{3}\left[\ell_{3}\right]}{\left(\ell_{1} \ell_{3}\right)^{\frac{1}{2}}} \sum_{(m n, q)=1} \sum_{(m n)^{\frac{1}{2}}} \frac{1}{\left(\frac{m n}{q}\right) \sum_{\chi(q)}^{+} \epsilon(\chi) \chi\left(m \ell_{1} \ell_{3}\right) \bar{\chi}(n) .}
\end{aligned}
$$


Opening $\epsilon(\chi)$ using (2.6) and applying Lemma 4.2, we find after some work (see [10, (3.4) and (3.7)]) that

$$
\sum_{\chi(q)}^{+} \epsilon(\chi) \chi\left(m \ell_{1} \ell_{3}\right) \bar{\chi}(n)=\frac{1}{q^{1 / 2}} \sum_{\substack{v w=q \\(v, w)=1}} \mu^{2}(v) \varphi(w) \cos \left(\frac{2 \pi n \overline{m \ell_{1} \ell_{3} v}}{w}\right) .
$$

The main term comes from $m \ell_{1} \ell_{3}=1$. With this constraint in place we apply character orthogonality in reverse, obtaining that the main term $M_{\mathrm{IS}, \mathrm{MV}}$ of Lemma 3.3 is

$$
M_{\mathrm{IS}, \mathrm{MV}}=2 P_{1}(1) P_{3}(1) \sum_{\chi(q)}^{+} \epsilon(\chi) \sum_{n} \frac{\bar{\chi}(n)}{n^{1 / 2}} V\left(\frac{n}{q}\right) .
$$

We have the following proposition.

Proposition 4.3. Let $\chi$ be a primitive even character modulo $q$, and let $T>0$ be a real number. Let $V$ be defined as in (4.1). Then

$$
\sum_{n} \frac{\bar{\chi}(n)}{n^{\frac{1}{2}}} V\left(\frac{T n}{q}\right)=L\left(\frac{1}{2}, \bar{\chi}\right)-\epsilon(\bar{\chi}) \sum_{n} \frac{\chi(n)}{n^{\frac{1}{2}}} F\left(\frac{n}{T}\right),
$$

where

$$
F(x)=\frac{1}{2 \pi i} \int_{(1)} \frac{\Gamma\left(\frac{s}{2}+\frac{1}{4}\right) \Gamma\left(-\frac{s}{2}+\frac{1}{4}\right)}{\Gamma^{2}\left(\frac{1}{4}\right)} \frac{G(s)}{s} x^{-s} d s .
$$

Before proving Proposition 4.3, let us see how to use it to finish the evaluation of $M_{\mathrm{IS}, \mathrm{MV}}$. Proposition 4.3 gives

$$
M_{\mathrm{IS}, \mathrm{MV}}=2 P_{1}(1) P_{3}(1) \sum_{\chi(q)}^{+} \epsilon(\chi) L\left(\frac{1}{2}, \bar{\chi}\right)-2 P_{1}(1) P_{3}(1) \sum_{\chi(q)}^{+} \sum_{n} \frac{\chi(n)}{n^{\frac{1}{2}}} F(n),
$$

and by the first moment analysis (see [12, section 3], also section 6 below) we have

$$
2 P_{1}(1) P_{3}(1) \sum_{\chi(q)}^{+} \epsilon(\chi) L\left(\frac{1}{2}, \bar{\chi}\right)=(1+o(1)) 2 P_{1}(1) P_{3}(1) \varphi^{+}(q) .
$$

For the other piece, we apply Lemma 4.2 to obtain

$$
-2 P_{1}(1) P_{3}(1) \sum_{\chi(q)}^{+} \sum_{n} \frac{\chi(n)}{n^{\frac{1}{2}}} F(n)=-P_{1}(1) P_{3}(1) \sum_{w \mid q} \varphi(w) \mu(q / w) \sum_{\substack{n \equiv \pm 1(w) \\(n, q)=1}} \frac{1}{n^{\frac{1}{2}}} F(n) .
$$

We choose $G$ to vanish at all the poles of

$$
\Gamma\left(\frac{s}{2}+\frac{1}{4}\right) \Gamma\left(-\frac{s}{2}+\frac{1}{4}\right)
$$

in the disc $|s| \leq A$, where $A>0$ is large but fixed. By moving the contour of integration to the right we see

$$
F(x) \ll \frac{1}{(1+x)^{100}},
$$

say, and therefore the contribution from $n>q^{\frac{1}{10}}$ is negligible. By trivial estimation the contribution from $w \leq q^{\frac{1}{4}}$ is also negligible. For $w>q^{\frac{1}{4}}$ and $n \leq q^{\frac{1}{10}}$, we can only have 
$n \equiv \pm 1(\bmod w)$ if $n=1$. Adding back in the terms with $n \leq q^{\frac{1}{4}}$, the contribution from these terms is therefore

$$
-(1+o(1)) 2 P_{1}(1) P_{3}(1) F(1) \varphi^{+}(q) .
$$

Since the integrand in $F(1)$ is odd, we may evaluate $F(1)$ through a residue at $s=0$. We shift the line of integration in (4.4) to $\operatorname{Re}(s)=-1$, picking up a contribution from the simple pole at $s=0$. In the integral on the line $\operatorname{Re}(s)=-1$ we change variables $s \rightarrow-s$. This yields the relation $F(1)=1-F(1)$, whence $F(1)=\frac{1}{2}$. Combining (4.5) and (4.7), we obtain

$$
M_{\mathrm{IS}, \mathrm{MV}}=(1+o(1)) P_{1}(1) P_{3}(1) \varphi^{+}(q),
$$

as desired. This yields the main term of Lemma 3.3 .

Proof of Proposition 4.3. We write $V$ using its definition and interchange orders of summation and integration to get

$$
\sum_{n} \frac{\bar{\chi}(n)}{n^{1 / 2}} V\left(\frac{T n}{q}\right)=\frac{1}{2 \pi i} \int_{(1)} \frac{\Gamma^{2}\left(\frac{s}{2}+\frac{1}{4}\right)}{\Gamma^{2}\left(\frac{1}{4}\right)} \frac{G(s)}{s}\left(\frac{q}{\pi}\right)^{s} T^{-s} L\left(\frac{1}{2}+s, \bar{\chi}\right) d s .
$$

We move the line of integration to $\operatorname{Re}(s)=-1$, picking up a contribution of $L\left(\frac{1}{2}, \bar{\chi}\right)$ from the pole at $s=0$. Observe that we do not get any contribution from the double pole of $\Gamma^{2}\left(\frac{s}{2}+\frac{1}{4}\right)$ at $s=-\frac{1}{2}$ because of our assumption that $G$ vanishes at $s= \pm \frac{1}{2}$ to second order.

Now, for the integral on the line $\operatorname{Re}(s)=-1$, we apply the functional equation for $L\left(\frac{1}{2}+s, \bar{\chi}\right)$ and then change variables $s \rightarrow-s$ to obtain

$$
-\epsilon(\bar{\chi}) \frac{1}{2 \pi i} \int_{(1)} \frac{\Gamma\left(\frac{s}{2}+\frac{1}{4}\right) \Gamma\left(-\frac{s}{2}+\frac{1}{4}\right)}{\Gamma^{2}\left(\frac{1}{4}\right)} \frac{G(s)}{s} T^{s} L\left(\frac{1}{2}+s, \chi\right) d s .
$$

The desired result follows by expanding $L\left(\frac{1}{2}+s, \chi\right)$ in its Dirichlet series and interchanging the order of summation and integration.

\section{LEMMA 3.3: ERROR TERM}

Here we show that the remainder of the terms in (4.2) (those with $m \ell_{1} \ell_{3} \neq 1$ ) contribute only to the error term of Lemma 3.3. Here we must avail ourselves of the averaging over $q$.

Inserting (4.3) into (4.2) and averaging over moduli, we wish to show that

$$
\begin{aligned}
& \mathcal{E}_{1}=\sum_{(v, w)=1} \sum^{2}(v) \frac{v}{\varphi(v)} \frac{w^{\frac{1}{2}}}{v^{\frac{1}{2}}} \Psi\left(\frac{v w}{Q}\right) \sum_{\substack{\ell_{1} \leq y_{1} \\
\ell_{3} \leq y_{3} \\
\left(\ell_{1} \ell_{3}, v w\right)=1}} \frac{\mu\left(\ell_{1}\right) \mu\left(\ell_{3}\right) P_{1}\left[\ell_{1}\right] P_{3}\left[\ell_{3}\right]}{\left(\ell_{1} \ell_{3}\right)^{\frac{1}{2}}} \\
& \times \sum_{(m n, v w)=1} \frac{1}{(m n)^{\frac{1}{2}}} Z\left(\frac{m n}{v w}\right) \cos \left(\frac{2 \pi n \overline{m \ell_{1} \ell_{3} v}}{w}\right) \ll Q^{2-\epsilon+o(1)},
\end{aligned}
$$

where $m \ell_{1} \ell_{3} \neq 1$, but we do not indicate this in the notation. The function $Z$ is actually just $V$ in (4.1), but we do not wish to confuse the function $V$ with the scale $V$ that shall appear shortly.

Observe that the arithmetic weight $\frac{q}{\varphi(q)}$ has become $\frac{v}{\varphi(v)} \frac{w}{\varphi(w)}$ by multiplicativity, and that this factor of $\varphi(w)$ has canceled with $\varphi(w)$ in (4.3), making the sum on $w$ smooth.

The main tool we use to bound $\mathcal{E}_{1}^{\prime}$ is the following result, due to Deshouillers and Iwaniec, on cancellation in sums of Kloosterman sums. 
Lemma 5.1. Let $C, D, N, R, S$ be positive numbers, and let $b_{n, r, s}$ be a complex sequence supported in $(0, N] \times(R, 2 R] \times(S, 2 S] \cap \mathbb{N}^{3}$. Let $g_{0}(\xi, \eta)$ be a smooth function having compact support in $\mathbb{R}^{+} \times \mathbb{R}^{+}$, and let $g(c, d)=g_{0}(c / C, d / D)$. Then

$$
\begin{aligned}
\sum_{c} \sum_{d} \sum_{\substack{n \\
(r d, s c)=1}} \sum_{r} \sum_{s} & b_{n, r, s} g(c, d) e\left(n \frac{\overline{r d}}{s c}\right) \\
& \ll_{\epsilon, g_{0}}(C D N R S)^{\epsilon} K(C, D, N, R, S)\left\|b_{N, R, S}\right\|_{2},
\end{aligned}
$$

where

$$
\left\|b_{N, R, S}\right\|_{2}=\left(\sum_{n} \sum_{r} \sum_{s}\left|b_{n, r, s}\right|^{2}\right)^{\frac{1}{2}}
$$

and

$$
K(C, D, N, R, S)^{2}=C S(R S+N)(C+R D)+C^{2} D S \sqrt{(R S+N) R}+D^{2} N R S^{-1} .
$$

Proof. This is essentially [3, Lemma 1], which is an easy consequence of [7, Theorem 12].

We need to massage (5.1) before it is in a form where an application of Lemma [5.1] is appropriate. Let us briefly describe our plan of attack. We apply partitions of unity to localize the variables and then separate variables with integral transforms. By using the orthogonality of multiplicative characters we will be able to assume that $v$ is quite small, which is advantageous when it comes time to remove coprimality conditions involving $v$. We next reduce to the case in which $n$ is somewhat small. This is due to the fact that the sum on $n$ is essentially a Ramanujan sum, and Ramanujan sums experience better than squareroot cancellation on average. With the size of $n$ suitably reduced, we may appeal to the reciprocity formula (5.7) and use Möbius inversion to remove the coprimality condition between $n$ and $w$. This application of Möbius inversion introduces a new variable, call if $f$, and another application of character orthogonality allows us to assume $f$ is small. We then remove the coprimality conditions on $m$. We finally apply Lemma 5.1 to get the desired cancellation, and it is crucial here that $f$ and $v$ are no larger than $Q^{\epsilon}$.

Let us turn to the details in earnest. We apply smooth partitions of unity (see [2, Lemma 1.6], for instance) in all variables, so that $\mathcal{E}_{1}$ can be written

$$
\sum_{M, N, L_{1}, L_{3}, V, W} \cdots \sum_{\mathcal{E}_{1}}\left(M, N, L_{1}, L_{3}, V, W\right)
$$

where

$$
\begin{aligned}
& \mathcal{E}_{1}\left(M, N, L_{1}, L_{3}, V, W\right)=\sum_{(v, w)=1} \sum_{\mu^{2}}(v) \frac{v}{\varphi(v)} \frac{w^{\frac{1}{2}}}{v^{\frac{1}{2}}} \Psi\left(\frac{v w}{Q}\right) G\left(\frac{v}{V}\right) G\left(\frac{w}{W}\right) \\
& \times \sum_{\substack{\ell_{1} \leq y_{1} \\
\ell_{3} \leq y_{3} \\
\left(\ell_{1} \ell_{3}, v w\right)=1}} \frac{\mu\left(\ell_{1}\right) \mu\left(\ell_{3}\right) P_{1}\left[\ell_{1}\right] P_{3}\left[\ell_{3}\right]}{\left(\ell_{1} \ell_{3}\right)^{\frac{1}{2}}} G\left(\frac{\ell_{1}}{L_{1}}\right) G\left(\frac{\ell_{3}}{L_{3}}\right) \\
& \times \sum_{(m n, v w)=1} \frac{1}{(m n)^{\frac{1}{2}}} Z\left(\frac{m n}{v w}\right) G\left(\frac{m}{M}\right) G\left(\frac{n}{N}\right) \cos \left(\frac{2 \pi n \overline{m \ell_{1} \ell_{3} v}}{w}\right) .
\end{aligned}
$$


Here $G$ is a smooth, nonnegative function supported in $\left[\frac{1}{2}, 2\right]$, and the numbers $M, N, L_{i}, V, W$ in (5.2) range over powers of two. We may assume

$$
M, N, L_{1}, L_{3}, V, W \gg 1, \quad V W \asymp Q, \quad L_{i} \ll y .
$$

Furthermore, by the rapid decay of $V$ we may assume $M N \leq Q^{1+\epsilon}$. Thus, the number of summands $\mathcal{E}_{1}(M, \ldots, W)$ in $(5.2)$ is $\ll Q^{o(1)}$.

Up to changing the definition of $G$, we may rewrite $\mathcal{E}_{1}(M, \cdots, W)$ as

$$
\begin{aligned}
\mathcal{E}_{1}\left(M, N, L_{1}, L_{3}, V, W\right) & =\frac{W^{\frac{1}{2}}}{\left(M N L_{1} L_{3} V\right)^{\frac{1}{2}}} \sum_{(v, w)=1} \alpha(v) G\left(\frac{v}{V}\right) G\left(\frac{w}{W}\right) \Psi\left(\frac{v w}{Q}\right) \\
& \times \sum_{\substack{\ell_{i} \leq y_{i} \\
\left(\ell_{i}, v w\right)=1}} \beta\left(\ell_{1}\right) \gamma\left(\ell_{3}\right) G\left(\frac{\ell_{1}}{L_{1}}\right) G\left(\frac{\ell_{3}}{L_{3}}\right) \\
& \times \sum_{(m n, v w)=1} \sum_{\substack{m w \\
v w}} Z\left(\frac{m}{M}\right) G\left(\frac{n}{N}\right) \cos \left(\frac{2 \pi n \overline{m \ell_{1} \ell_{3} v}}{w}\right),
\end{aligned}
$$

where $\alpha, \beta, \gamma$ are sequences satisfying $|\alpha(v)|,\left|\beta\left(\ell_{1}\right)\right|,\left|\gamma\left(\ell_{3}\right)\right| \ll Q^{o(1)}$.

We separate the variables in $Z$ by writing $Z$ using its definition as an integral (4.1) and moving the line of integration to $\operatorname{Re}(s)=L^{-1}$. By the rapid decay of the $\Gamma$ function in vertical strips we may restrict to $|\operatorname{Im}(s)| \leq Q^{\epsilon}$. We similarly separate the variables in $\Psi$ using the inverse Mellin transform. Therefore, up to changing the definition of some of the functions $G$, it suffices to prove that

$$
\begin{aligned}
\mathcal{E}_{1}^{\prime}\left(M, N, L_{1}, L_{3}, V, W\right) & =\frac{W^{\frac{1}{2}}}{\left(M N L_{1} L_{3} V\right)^{\frac{1}{2}}} \sum_{(v, w)=1} \alpha(v) G\left(\frac{v}{V}\right) G\left(\frac{w}{W}\right) \\
& \times \sum_{\substack{\ell_{i} \leq y_{i} \\
\left(\ell_{i}, v w\right)=1}} \beta\left(\ell_{1}\right) \gamma\left(\ell_{3}\right) G\left(\frac{\ell_{3}}{L_{3}}\right) G\left(\frac{\ell_{3}}{L_{3}}\right) \\
& \times \sum_{(m n, v w)=1} \sum_{\substack{m \\
M}} G\left(\frac{n}{N}\right) e\left(\frac{n \overline{m \ell_{1} \ell_{3} v}}{w}\right) \ll Q^{2-\epsilon+o(1)} .
\end{aligned}
$$

Our smooth functions $G$ all satisfy $G^{(j)}(x) \ll_{j} Q^{j \epsilon}$ for $j \geq 0$. To save on space we write the left side of (5.3) as simply $\mathcal{E}_{1}^{\prime}$.

Observe that the trivial bound for $\mathcal{E}_{1}^{\prime}$ is

$$
\mathcal{E}_{1}^{\prime} \ll V^{\frac{1}{2}} W^{\frac{3}{2}}(M N)^{\frac{1}{2}}\left(L_{1} L_{3}\right)^{\frac{1}{2}} Q^{o(1)} \ll \frac{Q^{2+\epsilon}\left(y_{1} y_{3}\right)^{\frac{1}{2}}}{V} .
$$

This bound is worst when $V$ is small. Since $y_{i}$ will be taken close to $Q^{\frac{1}{2}}$, we therefore need to save $\approx Q^{\frac{1}{2}}$ in order to obtain (5.1). The trivial bound does show, however, that the contribution from $V>Q^{\frac{1}{2}+2 \epsilon}$ is acceptably small, and we may therefore assume that $V \leq Q^{\frac{1}{2}+2 \epsilon}$. Note this implies $W \gg Q^{\frac{1}{2}-\epsilon}$. 
We now reduce to the case $V \ll Q^{\epsilon}$. We accomplish this by re-introducing multiplicative characters. The orthogonality of multiplicative characters yields

$$
e\left(\frac{n \overline{m \ell_{1} \ell_{3} v}}{w}\right)=\frac{1}{\varphi(w)} \sum_{\chi(w)} \tau(\bar{\chi}) \chi(n) \bar{\chi}\left(m \ell_{1} \ell_{3} v\right) .
$$

Using the Gauss sum bound $|\tau(\bar{\chi})| \ll w^{\frac{1}{2}}$ we then arrange $\mathcal{E}_{1}^{\prime}$ as

$$
\mathcal{E}_{1}^{\prime} \ll \frac{W}{\left(M N L_{1} L_{3} V\right)^{\frac{1}{2}}} \sum_{w \asymp W} \frac{1}{\varphi(w)} \sum_{v \asymp V}\left|\sum_{(m n, v)=1} \sum_{w} \chi(n) \bar{\chi}(m)\right|\left|\sum_{\left(\ell_{1} \ell_{3}, v\right)=1} \sum_{\chi}\left(\ell_{1} \ell_{3}\right)\right|,
$$

where we have suppressed some things in the notation for brevity. By Cauchy-Schwarz and character orthogonality we obtain

$$
\sum_{\chi(w)}\left|\sum_{m, n} \sum_{\mid}\right|\left|\sum_{\ell_{1}, \ell_{3}} \sum\right| \ll Q^{o(1)}\left(M N L_{1} L_{3}\right)^{\frac{1}{2}}(M N+W)^{\frac{1}{2}}\left(L_{1} L_{3}+W\right)^{\frac{1}{2}},
$$

which yields a bound of

$$
Q^{-o(1)} \mathcal{E}_{1}^{\prime} \ll \frac{Q(M N)^{\frac{1}{2}}\left(y_{1} y_{3}\right)^{\frac{1}{2}}}{V^{\frac{1}{2}}}+\frac{Q^{\frac{3}{2}}(M N)^{1 / 2}}{V}+\frac{Q^{\frac{3}{2}}\left(y_{1} y_{3}\right)^{\frac{1}{2}}}{V}+\frac{Q^{2}}{V^{\frac{3}{2}}} .
$$

We observe that (5.6) is acceptable for $V \geq Q^{3 \epsilon}$, say. We may therefore assume $V \leq Q^{\epsilon}$.

We next show that $\mathcal{E}_{1}^{\prime}$ is small provided $N$ is somewhat large.

Proposition 5.2. Assume the hypotheses of Lemma 3.3. If $N \geq M Q^{-2 \epsilon}$ and $m \ell_{1} \ell_{3} \neq 1$, then $\mathcal{E}_{1}^{\prime} \ll Q^{2-\epsilon+o(1)}$.

Proof. We make use only of cancellation in the sum on $n$, say

$$
\Sigma_{N}=\sum_{(n, v w)=1} G\left(\frac{n}{N}\right) e\left(\frac{n \overline{m \ell_{1} \ell_{3} v}}{w}\right) .
$$

We use Möbius inversion to detect the condition $(n, v)=1$, and then break $n$ into primitive residue classes modulo $w$. Thus

$$
\Sigma_{N}=\sum_{d \mid v} \mu(d) \sum_{(a, w)=1} e\left(\frac{a d \overline{m \ell_{1} \ell_{3} v}}{w}\right) \sum_{n \equiv a(w)} G\left(\frac{d n}{N}\right) .
$$

We apply Poisson summation to each sum on $n$, and obtain

$$
\Sigma_{N}=\sum_{d \mid v} \mu(d) \sum_{(a, w)=1} e\left(\frac{a d \overline{m \ell_{1} \ell_{3} v}}{w}\right) \frac{N}{d w} \sum_{|h| \leq W^{1+\epsilon} d / N} e\left(\frac{a h}{w}\right) \widehat{G}\left(\frac{h N}{d w}\right)+O_{\epsilon}\left(Q^{-100}\right),
$$

say. The contribution of the error term is, of course, negligible. The contribution of the zero frequency $h=0$ to $\Sigma_{N}$ is

$$
\widehat{G}(0) \frac{N}{w} \sum_{d \mid v} \frac{\mu(d)}{d} \sum_{(a, w)=1} e\left(\frac{a d \overline{m \ell_{1} \ell_{3} v}}{w}\right)=\widehat{G}(0) \mu(w) \frac{N}{w} \frac{\varphi(v)}{v},
$$

and upon summing this contribution over the remaining variables, the zero frequency contributes

$$
\ll V^{\frac{1}{2}} W^{\frac{1}{2}}(M N)^{\frac{1}{2}}\left(y_{1} y_{3}\right)^{\frac{1}{2}} Q^{o(1)} \ll Q^{\frac{3}{2}}
$$


to $\mathcal{E}_{1}^{\prime}$, and this contribution is sufficiently small.

It takes just a bit more work to bound the contribution of the nonzero frequencies $|h|>0$. We rearrange the sum as

$$
\sum_{d \mid v} \mu(d) \frac{N}{d w} \sum_{|h| \leq W^{1+\epsilon} d / N} \widehat{G}\left(\frac{h N}{d w}\right) \sum_{(a, w)=1} e\left(\frac{a d \overline{m \ell_{1} \ell_{3} v}}{w}+\frac{a h}{w}\right) .
$$

By a change of variables the inner sum is equal to the Ramanujan sum $c_{w}\left(h m \ell_{1} \ell_{3} v+d\right)$. Note that $h m \ell_{1} \ell_{3} v+d \neq 0$ because $m \ell_{1} \ell_{3} \neq 1$. The nonzero frequencies therefore contribute to $\mathcal{E}_{1}^{\prime}$ an amount

$$
\ll Q^{\epsilon} \frac{\left(V W L_{1} L_{3} M\right)^{\frac{1}{2}}}{N^{\frac{1}{2}}} \sup _{0<|k| \ll Q^{O(1)}} \sum_{w \asymp W}\left|c_{w}(k)\right| .
$$

Since $\left|c_{w}(k)\right| \leq(k, w)$ the sum on $w$ is $\ll W^{1+o(1)}$. It follows that

$$
\mathcal{E}_{1}^{\prime} \ll Q^{\frac{3}{2}}+Q^{\frac{3}{2}+\epsilon}\left(y_{1} y_{3}\right)^{\frac{1}{2}} \frac{M^{1 / 2}}{N^{\frac{1}{2}}} .
$$

Since $y_{i}=Q^{\theta_{i}}$ and $\theta_{i}<\frac{1}{2}-3 \epsilon$, say, this bound for $\mathcal{E}_{1}^{\prime}$ is acceptable provided $N \geq M Q^{-2 \epsilon}$.

By Proposition 5.2 we may assume $N \leq M Q^{-2 \epsilon}$. Since $M N \leq Q^{1+\epsilon}$, the condition $N \leq M Q^{-2 \epsilon}$ implies $N \leq Q^{\frac{1}{2}}$.

We now pause to make a comment on the condition $m \ell_{1} \ell_{3} \neq 1$, which we have assumed throughout this section but not indicated in the notation for $\mathcal{E}_{1}^{\prime}$. Observe that this condition is automatic if $M L_{1} L_{3}>2018$. If $M L_{1} L_{3} \ll 1$, then we may use the trivial bound (5.4) along with the bound $N \leq Q^{\frac{1}{2}} \leq Q^{1-\epsilon}$ to obtain

$$
\mathcal{E}_{1}^{\prime} \ll Q^{2-\epsilon} \text {. }
$$

We may therefore assume $M L_{1} L_{2} \gg 1$, so that the condition $m \ell_{1} \ell_{3} \neq 1$ is satisfied.

Since $N$ is relatively small, we may profitably apply the reciprocity formula

$$
\frac{\bar{x}}{y}+\frac{\bar{y}}{x} \equiv \frac{1}{x y}(\bmod 1)
$$

which holds for coprime integers $x, y$. Thus

$$
\frac{\overline{m \ell_{1} \ell_{3} v}}{w} \equiv-\frac{\bar{w}}{m \ell_{1} \ell_{3} v}+\frac{1}{m \ell_{1} \ell_{3} v w} \quad(\bmod 1)
$$

and $\mathcal{E}_{1}^{\prime}$ becomes

$$
\begin{aligned}
& \frac{W^{\frac{1}{2}}}{\left(M N L_{1} L_{3} V\right)^{\frac{1}{2}}} \sum_{(v, w)=1} \alpha(v) G\left(\frac{v}{V}\right) G\left(\frac{w}{W}\right) \sum_{\substack{\ell_{i} \leq y_{i} \\
\left(\ell_{i}, v w\right)=1}} \beta\left(\ell_{1}\right) \gamma\left(\ell_{3}\right) G\left(\frac{\ell_{1}}{L_{1}}\right) G\left(\frac{\ell_{3}}{L_{3}}\right) \\
& \times \sum_{(m n, v w)=1} G\left(\frac{m}{M}\right) G\left(\frac{n}{N}\right) e\left(-\frac{n \bar{w}}{m \ell_{1} \ell_{3} v}\right) e\left(\frac{n}{m \ell_{1} \ell_{3} v w}\right) .
\end{aligned}
$$

Observe that

$$
\frac{n}{m \ell_{1} \ell_{3} v w} \ll \frac{N}{M L_{1} L_{3} V W} \ll \frac{N}{Q} \ll \frac{1}{Q^{\frac{1}{2}}}=o(1),
$$


so by Taylor expansion we may write

$$
e\left(\frac{n}{m \ell_{1} \ell_{3} v w}\right)=1+O\left(\frac{N}{M L_{1} L_{3} V W}\right) .
$$

The contribution of this error term to $\mathcal{E}_{1}^{\prime}$ is

$$
\ll Q^{o(1)} \frac{W^{\frac{1}{2}} N^{\frac{3}{2}}}{\left(M L_{1} L_{3} V\right)^{\frac{1}{2}}} \ll W^{\frac{1}{2}+o(1)} N^{\frac{3}{2}} \ll Q^{2-\epsilon},
$$

and this is acceptably small. Therefore

$$
\begin{aligned}
\mathcal{E}_{1}^{\prime}=O\left(Q^{2-\epsilon}\right)+\frac{W^{\frac{1}{2}}}{\left(M N L_{1} L_{3} V\right)^{\frac{1}{2}}} & \sum_{(v, w)=1} \alpha(v) G\left(\frac{v}{V}\right) G\left(\frac{w}{W}\right) \\
& \times \sum_{\substack{\ell_{i} \leq y_{i} \\
\left(\ell_{i}, v w\right)=1}} \beta\left(\ell_{1}\right) \gamma\left(\ell_{3}\right) G\left(\frac{\ell_{1}}{L_{1}}\right) G\left(\frac{\ell_{3}}{L_{3}}\right) \\
& \times \sum_{(m n, v w)=1} \sum_{\substack{m \\
M}} G\left(\frac{n}{N}\right) e\left(-\frac{n \bar{w}}{m \ell_{1} \ell_{3} v}\right) .
\end{aligned}
$$

With $\mathcal{E}_{1}^{\prime}$ in this form we are in position to remove the coprimality condition $(n, w)=1$. By Möbius inversion we have

$$
\mathbf{1}_{(n, w)=1}=\sum_{\substack{f|n \\ f| w}} \mu(f) .
$$

We move the sum on $f$ to be the outermost sum, and note $f \ll N$. We then change variables $n \rightarrow n f, w \rightarrow w f$ and observe that

$$
\frac{n f \overline{w f}}{m \ell_{1} \ell_{3} v} \equiv \frac{n \bar{w}}{m \ell_{1} \ell_{3} v} \quad(\bmod 1) .
$$

By another application of (5.7) we find, after bounding an error term, that

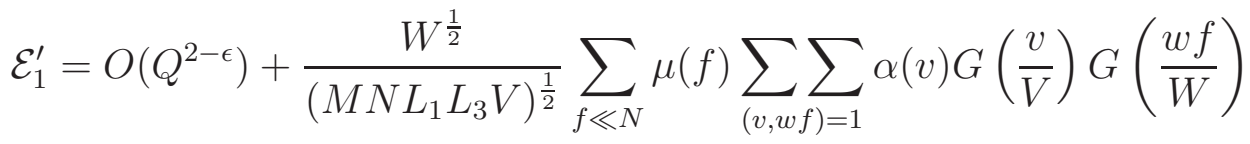

$$
\begin{aligned}
& \times \sum_{\substack{\ell_{i} \leq y_{i} \\
\left(\ell_{i}, f v w\right)=1}} \beta\left(\ell_{1}\right) \gamma\left(\ell_{3}\right) G\left(\frac{\ell_{1}}{L_{1}}\right) G\left(\frac{\ell_{3}}{L_{3}}\right) \sum_{\substack{(m, f v w)=1 \\
(n, v)=1}} \sum_{\substack{m \\
M}} G\left(\frac{n f}{N}\right) e\left(\frac{n \overline{m \ell_{1} \ell_{3} v}}{w}\right) .
\end{aligned}
$$

We next reduce the size of $f$ by a similar argument to the one that let us impose the condition $V \leq Q^{\epsilon}$. We obtain by transitioning to multiplicative characters (recall (5.5)) that the sum over $v, w, m, n, \ell_{1}, \ell_{3}$ is bounded by

$$
\ll \frac{W^{\frac{1}{2}+o(1)} V^{\frac{1}{2}}}{f^{\frac{1}{2}}} \sum_{w \asymp W / f} \frac{1}{w^{\frac{1}{2}}}\left(\frac{(M N)^{\frac{1}{2}}}{f^{\frac{1}{2}}}+w^{\frac{1}{2}}\right)\left(\left(L_{1} L_{3}\right)^{\frac{1}{2}}+w^{\frac{1}{2}}\right) \ll \frac{Q^{2+\epsilon}}{f^{\frac{3}{2}}},
$$

and therefore the contribution from $f>Q^{4 \epsilon}$ is negligible. 
Now the only barrier to applying Lemma 5.1 is the conditions $(m, f)=1$ and $(m, v)=1$. We remove both of these conditions with Möbius inversion, obtaining

$$
\begin{aligned}
& \sum_{f \ll \min \left(N, Q^{\epsilon}\right)} \mu(f) \sum_{h \mid f} \mu(h) \sum_{t \ll V} \mu(t) \frac{W^{\frac{1}{2}}}{\left(M N L_{1} L_{3} V\right)^{\frac{1}{2}}} \sum_{\substack{(v, w f)=1 \\
(w, h t)=1}} \alpha(v) G\left(\frac{v t}{V}\right) G\left(\frac{w f}{W}\right) \\
& \times \sum_{\substack{\ell_{i} \leq y_{i} \\
\left(\ell_{i}, f v w\right)=1}} \beta\left(\ell_{1}\right) \gamma\left(\ell_{3}\right) G\left(\frac{\ell_{1}}{L_{1}}\right) G\left(\frac{\ell_{3}}{L_{3}}\right) \sum_{\substack{(m, w)=1 \\
(n, v)=1}} \sum_{\substack{m \\
M}} G\left(\frac{n f}{N}\right) e\left(\frac{n \overline{m h t^{2} \ell_{1} \ell_{3} v}}{w}\right) .
\end{aligned}
$$

We set

$$
b_{n, h t^{2} k}=\mathbf{1}_{(n, v)=1} G\left(\frac{n f}{N}\right) \sum_{\substack{\ell_{1} \\ \ell_{1} \ell_{3} v=k \\\left(\ell_{1} \ell_{3}, v\right)=1}} \sum_{v} \beta\left(\ell_{1}\right) \gamma\left(\ell_{3}\right) \alpha(v) G\left(\frac{v t}{V}\right) G\left(\frac{\ell_{1}}{L_{1}}\right) G\left(\frac{\ell_{3}}{L_{3}}\right)
$$

if $(k, f)=1$, and for integers $r$ not divisible by $h t^{2}$ we set $b_{n, r}=0$. It follows that if $b_{n, r} \neq 0$, then $n \asymp N / f$ and $r \asymp h t L_{1} L_{3} V$ with $r \equiv 0\left(h t^{2}\right)$. The sum over $n, r, m, w$ is therefore a sum of the form to which Lemma 5.1 may be applied. We note that

$$
\left\|b_{N, R}\right\|_{2} \ll \frac{Q^{o(1)}}{(f t)^{\frac{1}{2}}}\left(N L_{1} L_{3} V\right)^{\frac{1}{2}},
$$

and therefore by Lemma 5.1 we have

$$
\begin{aligned}
\mathcal{E}_{1}^{\prime} & \ll Q^{\epsilon} \sum_{f \ll Q^{\epsilon}} \frac{1}{f^{\frac{1}{2}}} \sum_{h \mid f} \sum_{t \ll Q^{\epsilon}} \frac{1}{t^{\frac{1}{2}}} \frac{W^{\frac{1}{2}}}{M^{\frac{1}{2}}} \\
& \times\left\{\frac{W^{\frac{1}{2}}}{f^{\frac{1}{2}}}\left(\left(h t L_{1} L_{3} V\right)^{\frac{1}{2}}+\frac{N^{\frac{1}{2}}}{f^{\frac{1}{2}}}\right)\left(\frac{W^{\frac{1}{2}}}{f^{\frac{1}{2}}}+\left(M L_{1} L_{3} V\right)^{\frac{1}{2}}\right)\right. \\
& \left.+\frac{W}{f} \frac{M^{\frac{1}{2}}}{(h t)^{\frac{1}{2}}}\left(\left(h t L_{1} L_{3} V\right)^{\frac{1}{2}}+\left(h t L_{1} L_{3} N V\right)^{\frac{1}{4}}\right)+\frac{M}{h t}\left(h t L_{1} L_{3} N V\right)^{\frac{1}{2}}\right\} \\
& \ll Q^{\epsilon}\left(\frac{W^{\frac{3}{2}}\left(y_{1} y_{3}\right)^{\frac{1}{2}}}{M^{\frac{1}{2}}}+W y_{1} y_{3}+W^{\frac{3}{2}} \frac{N^{\frac{1}{2}}}{M^{\frac{1}{2}}}+W\left(y_{1} y_{3}\right)^{\frac{1}{2}} N^{\frac{1}{2}}+W^{\frac{3}{2}}\left(y_{1} y_{3}\right)^{\frac{1}{2}}\right. \\
& \left.+W^{\frac{3}{2}}\left(y_{1} y_{3}\right)^{\frac{1}{4}} N^{\frac{1}{4}}+W^{\frac{1}{2}} Q^{\frac{1}{2}}\left(y_{1} y_{3}\right)^{\frac{1}{2}}\right) \ll Q^{2-\epsilon},
\end{aligned}
$$

upon recalling the bounds $W \ll Q, y_{i} \leq Q^{\theta_{i}}$ with $\theta_{i}<\frac{1}{2}$, and $N \leq Q^{1-\epsilon}$. This completes the proof of Lemma 3.3.

\section{LEMMa 3.4; MAIN TERM}

In this section we obtain the main term of Lemma 3.4. We allow ourselves to recycle some notation from sections 4 and 5 . 
Recall that we wish to asymptotically evaluate

$$
\sum_{\chi(q)}^{+}\left|L\left(\frac{1}{2}, \chi\right)\right|^{2} \psi_{\mathrm{B}}(\chi) \psi_{\mathrm{MV}}(\bar{\chi})
$$

We begin precisely as in section 4 . Inserting the definitions of $\psi_{\mathrm{B}}(\chi)$ and $\psi_{\mathrm{MV}}(\bar{\chi})$, we must asympotically evaluate

$$
\frac{2}{L} \sum_{\substack{b c \leq y_{2} \\(b c, q)=1}} \frac{\Lambda(b) \mu(c) P_{2}[b c]}{(b c)^{\frac{1}{2}}} \sum_{\substack{\ell \leq y_{3} \\(\ell, q)=1}} \frac{\mu(\ell) P_{3}[\ell]}{\ell^{\frac{1}{2}}} \sum_{(m n, q)=1} \sum_{(m n)^{\frac{1}{2}}} V\left(\frac{m n}{q}\right) \sum_{\chi(q)}^{+} \epsilon(\chi) \chi(c \ell m) \bar{\chi}(b n) .
$$

The main term of Lemma 3.3 arose from $m \ell_{1} \ell_{3}=1$. In the present case, the main term contains more than just $c \ell m=1$; the main term arises from those $c \ell m$ which divide $b$. The support of the von Mangoldt function constrains $b$ to be a prime power, so the condition $c \ell m \mid b$ is straightforward, but tedious, to handle.

There are three different cases to consider. The first case is $c l m=1$. In the second case we have $c \ell m=p$ and $b=p$. Both of these cases contribute to the main term. The third case is everything else $\left(b=p^{j}\right.$ with $j \geq 2$ and $c \ell m \mid b$ with $\left.c l m \geq p\right)$, and this case contributes only to the error term.

\subsection{First case: $c l m=1$.}

If $c \ell m$ is equal to 1 then certainly $c \ell m$ divides $b$ for every $b$. The contribution from $c \ell m=1$ is equal to

$$
M=\frac{2 P_{3}(1)}{L} \sum_{\chi(q)}^{+} \epsilon(\chi) \sum_{b \leq y_{2}} \frac{\Lambda(b) \bar{\chi}(b) P_{2}[b]}{b^{\frac{1}{2}}} \sum_{n} \frac{\bar{\chi}(n)}{n^{\frac{1}{2}}} V\left(\frac{n}{q}\right) .
$$

By an application of Proposition 4.3,

$$
M=M_{1}+M_{2},
$$

where

$$
\begin{aligned}
& M_{1}=\frac{2 P_{3}(1)}{L} \sum_{\substack{b \leq y_{2} \\
(b, q)=1}} \frac{\Lambda(b) P_{2}[b]}{b^{\frac{1}{2}}} \sum_{\chi(q)}^{+} \epsilon(\chi) \bar{\chi}(b) L\left(\frac{1}{2}, \bar{\chi}\right), \\
& M_{2}=-\frac{2 P_{3}(1)}{L} \sum_{\substack{b \leq y_{2} \\
(b n, q)=1}} \frac{\Lambda(b) P_{2}[b]}{(b n)^{\frac{1}{2}}} F(n) \sum_{\chi(q)}^{+} \chi(n) \bar{\chi}(b),
\end{aligned}
$$

and $F$ is the rapidly decaying function given by (4.4). A main term arises from $M_{1}$, and $M_{2}$ contributes only to the error term.

Let us first investigate $M_{2}$. By Lemma 4.2 we have

$$
M_{2}=-\frac{P_{3}(1)}{L} \sum_{w \mid q} \varphi(w) \mu(q / w) \sum_{\substack{b \leq y_{2} \\ b \equiv \pm n(w) \\(b n, q)=1}} \frac{\Lambda(b) P_{2}[b]}{(b n)^{\frac{1}{2}}} F(n) .
$$


By the rapid decay of $F$ (recall (4.6) ) we may restrict $n$ to $n \leq q^{\frac{1}{10}}$. The contribution from $w \leq q^{\frac{1}{2}+\epsilon}$ is then trivially $\ll q^{1-\epsilon}$, since $y_{2} \ll q^{\frac{1}{2}-\epsilon}$. For the remaining terms, the congruence condition $b \equiv \pm n(w)$ becomes $b=n$, and thus

$$
M_{2} \ll q^{1-\epsilon}+\frac{1}{L} \sum_{\substack{w \mid q \\ w>q^{\frac{1}{2}+\epsilon}}} \varphi(w) \sum_{b \leq q^{\frac{1}{10}}} \frac{\Lambda(b) P_{2}[b]}{b} F(b) \ll q L^{-1} .
$$

Let us turn to $M_{1}$. We use the following lemma to represent the central value $L\left(\frac{1}{2}, \bar{\chi}\right)$.

Lemma 6.1. Let $\bar{\chi}$ be a primitive even character modulo $q$. Then

$$
L\left(\frac{1}{2}, \bar{\chi}\right)=\sum_{n} \frac{\bar{\chi}(n)}{n^{\frac{1}{2}}} V_{1}\left(\frac{n}{q^{\frac{1}{2}}}\right)+\epsilon(\bar{\chi}) \sum_{n} \frac{\chi(n)}{n^{\frac{1}{2}}} V_{1}\left(\frac{n}{q^{\frac{1}{2}}}\right),
$$

where

$$
V_{1}(x)=\frac{1}{2 \pi i} \int_{(1)} \frac{\Gamma\left(\frac{s}{2}+\frac{1}{4}\right)}{\Gamma\left(\frac{1}{4}\right)} \frac{G_{1}(s)}{s} \pi^{-s / 2} x^{-s} d s
$$

and $G_{1}(s)$ is an even polynomial satisfying $G_{1}(0)=1$.

Proof. See [10, (2.2)].

Applying Lemma 6.1, the main term $M_{1}$ naturally splits as $M_{1}=M_{1,1}+M_{1,2}$, where

$$
\begin{aligned}
& M_{1,1}=\frac{2 P_{3}(1)}{L} \sum_{\substack{b \leq y_{2} \\
(b n, q)=1}} \frac{\Lambda(b) P_{2}[b]}{(b n)^{\frac{1}{2}}} V_{1}\left(\frac{n}{q^{\frac{1}{2}}}\right) \sum_{\chi(q)}^{+} \epsilon(\chi) \bar{\chi}(b n), \\
& M_{1,2}=\frac{2 P_{3}(1)}{L} \sum_{\substack{b \leq y_{2} \\
(b n, q)=1}} \frac{\Lambda(b) P_{2}[b]}{(b n)^{\frac{1}{2}}} V_{1}\left(\frac{n}{q^{\frac{1}{2}}}\right) \sum_{\chi(q)}^{+} \chi(n) \bar{\chi}(b) .
\end{aligned}
$$

Applying character orthogonality to $M_{1,1}$ we arrive at

$$
M_{1,1}=\frac{2 P_{3}(1)}{L q^{1 / 2}} \sum_{\substack{v w=q \\(v, w)=1}} \mu^{2}(v) \varphi(w) \sum_{\substack{b \leq y_{2} \\(b n, q)=1}} \frac{\Lambda(b) P_{2}[b]}{(b n)^{\frac{1}{2}}} V_{1}\left(\frac{n}{q^{\frac{1}{2}}}\right) \cos \left(\frac{2 \pi b n \bar{v}}{w}\right),
$$

and a trivial estimation shows

$$
M_{1,1} \ll q^{1-\epsilon} .
$$

Let us lastly examine $M_{1,2}$, from which a main term arises. By character orthogonality we have

$$
M_{1,2}=\frac{P_{3}(1)}{L} \sum_{w \mid q} \varphi(w) \mu(q / w) \sum_{\substack{b \leq y_{2} \\ b \equiv \pm n(w) \\(b, q)=1}} \frac{\Lambda(b) P_{2}[b]}{(b n)^{\frac{1}{2}}} V_{1}\left(\frac{n}{q^{\frac{1}{2}}}\right)
$$

By trivial estimation, the contribution from $w \leq q^{\frac{1}{2}+\epsilon}$ is

$$
\ll \sum_{\substack{w \mid q \\ w \leq q^{\frac{1}{2}+\epsilon}}} \varphi(w) \sum_{b \leq y_{2}} \frac{1}{b^{1 / 2}} \sum_{\substack{n \leq q^{\frac{1}{2}+\epsilon} \\ n \equiv \pm b(w)}} \frac{1}{n^{\frac{1}{2}}} \ll y_{2}^{\frac{1}{2}} \sum_{\substack{w \mid q \\ w \leq q^{\frac{1}{2}+\epsilon}}} \varphi(w)\left(\frac{q^{\frac{1}{4}+\epsilon}}{w}+O(1)\right) \ll q^{\frac{3}{4}+\epsilon} .
$$


By the rapid decay of $V_{1}$, for $w>q^{\frac{1}{2}+\epsilon}$ the congruence $b \equiv \pm n(w)$ becomes $b=n$. Adding back in the terms $w \leq q^{\frac{1}{2}+\epsilon}$, we have

$$
M_{1,2}=\frac{2 P_{3}(1)}{L} \varphi^{+}(q) \sum_{\substack{b \leq y_{2} \\(b, q)=1}} \frac{\Lambda(b) P_{2}[b]}{b} V_{1}\left(\frac{b}{q^{\frac{1}{2}}}\right)+O\left(q^{1-\epsilon}\right) .
$$

For $x \ll 1$ we see by a contour shift that

$$
V_{1}(x)=1+O\left(x^{\frac{1}{3}}\right),
$$

and we have $b q^{-1 / 2} \ll q^{-\epsilon}$. It follows that

$$
M_{1,2}=O\left(q^{1-\epsilon}\right)+\frac{2 P_{3}(1)}{L} \varphi^{+}(q) \sum_{\substack{b \leq y_{2} \\(b, q)=1}} \frac{\Lambda(b) P_{2}[b]}{b} .
$$

We have

$$
\sum_{(b, q)>1} \frac{\Lambda(b)}{b} \ll 1+\sum_{p \mid q} \frac{\log p}{p} \ll \log \log q,
$$

and therefore we may remove the condition $(b, q)=1$ at the cost of an error $O\left(q L^{-1+\epsilon}\right)$. From the estimate

$$
\sum_{n \leq x} \frac{\Lambda(n)}{n}=\log x+O(1),
$$

summation by parts, and elementary manipulations, we obtain

$$
\sum_{b \leq y_{2}} \frac{\Lambda(b) P_{2}[b]}{b}=\left(\log y_{2}\right) \int_{0}^{1} P_{2}(u) d u+O(1) .
$$

Therefore, the contribution to the main term of Lemma 3.4 from $c l m=1$ is

$$
\left(2 \theta_{2} P_{3}(1) \widetilde{P_{2}}(1)+o(1)\right) \varphi^{+}(q) .
$$

Second case: $c l m=p, b=p$.

Another main term which contributes to Lemma 3.4 comes from $c \ell m=p$ and $b=p$. There are three subcases: $(c, \ell, m)=(p, 1,1),(1, p, 1)$, or $(1,1, p)$. These three cases give (compare with (6.1) )

$$
\begin{aligned}
& N_{1}=-\frac{2 P_{3}(1)}{L} \sum_{\substack{p \leq y_{2}^{1 / 2} \\
(p, q)=1}} \frac{(\log p) P_{2}\left(\frac{\log \left(y_{2}^{1 / 2} / p\right)}{\log \left(y_{2}^{1 / 2}\right)}\right)}{p} \sum_{\chi(q)}^{+} \epsilon(\chi) \sum_{n} \frac{\bar{\chi}(n)}{n^{\frac{1}{2}}} V\left(\frac{n}{q}\right), \\
& N_{2}=-\frac{2}{L} \sum_{\substack{p \leq y_{2} \\
(p, q)=1}} \frac{(\log p) P_{2}[p] P_{3}[p]}{p} \sum_{\chi(q)}^{+} \epsilon(\chi) \sum_{n} \frac{\bar{\chi}(n)}{n^{\frac{1}{2}}} V\left(\frac{n}{q}\right), \\
& N_{3}=\frac{2 P_{3}(1)}{L} \sum_{\substack{p \leq y_{2} \\
(p, q)=1}} \frac{(\log p) P_{2}[p]}{p} \sum_{\chi(q)}^{+} \epsilon(\chi) \sum_{n} \frac{\bar{\chi}(n)}{n^{\frac{1}{2}}} V\left(\frac{p n}{q}\right) .
\end{aligned}
$$


The first two are somewhat easier to handle than the last one. We apply Proposition 4.3 then argue as in section 4 and the $c \ell m=1$ case to obtain

$$
\sum_{\chi(q)}^{+} \epsilon(\chi) \sum_{n} \frac{\bar{\chi}(n)}{n^{1 / 2}} V\left(\frac{n}{q}\right)=\frac{1}{2} \varphi^{+}(q)+O\left(q^{1-\epsilon}\right) .
$$

It follows that

$$
\begin{aligned}
& N_{1}=-\left(\frac{\theta_{2}}{2} P_{3}(1) \widetilde{P_{2}}(1)+o(1)\right) \varphi^{+}(q) \\
& N_{2}=-\left(\theta_{2} \int_{0}^{1} P_{2}(u) P_{3}(u) d u+o(1)\right) \varphi^{+}(q) .
\end{aligned}
$$

Combining (6.3) and (6.4) gives the main term of Lemma 3.4.

The final term $N_{3}$ is more difficult because the inner sum now depends on $p$. However, $M_{3}$ contributes only to the error term. By Proposition 4.3 with $T=p$,

$$
\sum_{n} \frac{\bar{\chi}(n)}{n^{\frac{1}{2}}} V\left(\frac{p n}{q}\right)=L\left(\frac{1}{2}, \bar{\chi}\right)-\sum_{n} \frac{\chi(n)}{n^{\frac{1}{2}}} F\left(\frac{n}{p}\right) .
$$

The first term on the right side of (6.5) contributes to $N_{3}$ an amount

$$
\left(2 \theta_{2} P_{3}(1) \widetilde{P_{2}}(1)+o(1)\right) \varphi^{+}(q)
$$

For the second term on the right side of (6.5) we use character orthogonality and get

$$
-\frac{2 P_{3}(1)}{L} \sum_{\substack{p \leq y_{2} \\(p, q)=1}} \frac{(\log p) P_{2}[p]}{p} \frac{1}{2} \sum_{w \mid q} \varphi(w) \mu(q / w) \sum_{n \equiv \pm 1(w)} \frac{1}{n^{\frac{1}{2}}} F\left(\frac{n}{p}\right) .
$$

By the rapid decay of $F$ the contribution from $n>p^{\frac{11}{10}}$, say, is $O\left(q L^{-1}\right)$. We next estimate trivially the contribution from $w \leq q^{\frac{3}{5}}$, say. We have the bound

$$
\sum_{\substack{n \equiv \pm 1(w) \\ n \leq p \frac{11}{10}}} \frac{1}{n^{\frac{1}{2}}} F\left(\frac{n}{p}\right) \ll q^{\epsilon}\left(\frac{p^{\frac{11}{20}}}{w}+1\right),
$$

and this contributes to $N_{3}$ an amount

$$
\ll q^{\frac{3}{5}+\epsilon}+q^{\epsilon} \sum_{p \leq y_{2}} p^{-\frac{9}{20}} \ll q^{\frac{3}{5}+\epsilon},
$$

since $y_{2} \ll q^{\frac{1}{2}}$. For $w>q^{\frac{3}{5}}$ and $n \leq p^{\frac{11}{10}}$ the congruence $n \equiv \pm 1(w)$ becomes $n=1$. By a contour shift we have

$$
F\left(\frac{1}{p}\right)=1+O\left(p^{-\frac{1}{2}}\right)
$$

Thus, the second term on the right side of (6.5) contributes to $N_{3}$ an amount

$$
-\left(2 \theta_{2} P_{3}(1) \widetilde{P_{2}}(1)+o(1)\right) \varphi^{+}(q)
$$

and (6.6) and (6.7) together imply $N_{3}$ is negligible. 


\section{Third case: everything else.}

This case is the contribution from $b=p^{j}$ with $j \geq 2$ and $c \ell m \mid b$ with $c \ell m \geq p$. This case contributes an error of size $O\left(q L^{-1+\epsilon}\right)$, essentially because the sum

$$
\sum_{\substack{p^{k} \\ k \geq 2}} \frac{\log \left(p^{k}\right)}{p^{k}}
$$

converges. There are four different subcases to consider, since the Möbius functions attached to $c$ and $\ell$ imply $c, \ell \in\{1, p\}$. The same techniques we have already employed allow one to bound the resulting sums, so we leave the details for the interested reader. This completes the proof of Lemma 3.4 .

\section{LEMMA 3.4: ERROR TERM}

After the results of the previous section, it remains to finish the proof of Lemma 3.4 by showing the error term of (6.1) is negligible. The argument is very similar to that given in section 5, and, indeed, the arguments are identical after a point.

The error term has the form

$$
\begin{aligned}
\mathcal{E}_{2} & =\sum_{(v, w)=1} \sum^{2}(v) \frac{v}{\varphi(v)} \frac{w^{\frac{1}{2}}}{v^{\frac{1}{2}}} \Psi\left(\frac{v w}{Q}\right) \sum_{\substack{\ell \leq y_{3} \\
(\ell, v w)=1}} \frac{\mu(\ell) P_{3}[\ell]}{\ell^{\frac{1}{2}}} \\
& \times \sum_{\substack{b c \leq y_{2} \\
(b c, v w)=1}} \frac{\Lambda(b) \mu(c) P_{2}[b c]}{(b c)^{\frac{1}{2}}} \sum_{(m n, v w)=1} \sum_{\frac{1}{(m n)^{\frac{1}{2}}}} V\left(\frac{m n}{v w}\right) \cos \left(\frac{2 \pi b n \overline{c \ell m v}}{w}\right),
\end{aligned}
$$

where we also have the condition $c \ell m \nmid b$, which we do not indicate in the notation. This condition is awkward, but turns out to be harmless.

We note that we may separate the variables $b$ and $c$ from one another in $P_{2}[b c]$ by linearity, the additivity of the logarithm, and the binomial theorem. Thus, it suffices to study $\mathcal{E}_{1}$ with $P_{2}[b c]$ replaced by $(\log b)^{j_{1}}(\log c)^{j_{2}}$, for $j_{i}$ some fixed nonnegative integers. Arguing as in the reduction to (5.3), we may bound $\mathcal{E}_{2}$ by $\ll Q^{o(1)}$ instances of $\mathcal{E}_{2}^{\prime}=\mathcal{E}_{2}^{\prime}(B, C, L, M, N, V, W)$, where

$$
\begin{aligned}
\mathcal{E}_{2}^{\prime} & =\frac{W^{\frac{1}{2}}}{(B C L M N V)^{\frac{1}{2}}} \sum_{(v, w)=1} \sum \alpha(v) G\left(\frac{v}{V}\right) G\left(\frac{w}{W}\right) \\
& \times \sum_{\substack{\ell \leq y_{3} \\
(\ell, v w)=1}} \beta(\ell) G\left(\frac{\ell}{L}\right) \sum_{\substack{b c \leq y_{2} \\
(b c, v w)=1}} \gamma(b) \delta(c) G\left(\frac{b}{B}\right) G\left(\frac{c}{C}\right) \\
& \times \sum_{(m n, v w)=1} G\left(\frac{m}{M}\right) G\left(\frac{n}{N}\right) e\left(\frac{b n \overline{c \ell m v}}{w}\right),
\end{aligned}
$$

the function $G$ is smooth as before, and $\alpha, \beta, \gamma, \delta$ are sequences $f$ satisfying $|f(z)| \ll Q^{o(1)}$. We also have the conditions

$$
V W \asymp Q, \quad M N \leq Q^{1+\epsilon}, \quad B C \ll y_{2}, \quad L \ll y_{3}, \quad B, C, L, M, N, V, W \gg 1 .
$$


By the argument that gave (5.6) we may also assume $V \leq Q^{\epsilon}$. Lastly, we may remove the condition $b c \leq y_{2}$ by Mellin inversion, at the cost of changing $\gamma$ and $\delta$ by $b^{i t_{0}}, c^{i t_{0}}$, respectively, where $t_{0} \in \mathbb{R}$ is arbitrary (see [8, Lemma 9], for instance).

Recall the condition $c \ell m \nmid b$. This condition is unnecessary if $C L M>2018 B$, so it is only in the case $C L M \ll B$ where we need to deal with it. However, the case $C L M \ll B$ is exceptional, since $B$ is bounded by $y_{2} \ll Q^{\frac{1}{2}}$ but generically we would expect $C L M$ to be much larger than $Q^{\frac{1}{2}}$.

Indeed, we now show that when $C L M \ll B$ it suffices to get cancellation from the $n$ variable alone. The proof is essentially Proposition 5.2, so we just remark upon the differences. By Möbius inversion and Poisson summation we have

$$
\begin{aligned}
\sum_{(n, v w)=1} G\left(\frac{n}{N}\right) e\left(\frac{b n \overline{c \ell m v}}{w}\right) & =\mu(w) \frac{N}{w} \frac{\varphi(v)}{v} \\
& +\sum_{d \mid v} \mu(d) \frac{N}{d w} \sum_{|h| \leq W^{1+\epsilon} d / N} \widehat{G}\left(\frac{h N}{d w}\right) \sum_{(a, w)=1} e\left(\frac{a b d \overline{c \ell m v}}{w}+\frac{a h}{w}\right) \\
& +O\left(Q^{-100}\right) .
\end{aligned}
$$

The first and third terms contribute acceptable amounts, so consider the second term. The sum over $a$ is the Ramanujan sum $c_{w}(h c l m v+b d)$, and since $c l m$ does not divide $b$ the argument of the Ramanujan sum is non-zero. Following the proof of Proposition 5.2, we therefore obtain a bound of

$$
\mathcal{E}_{2}^{\prime} \ll \frac{Q^{\frac{3}{2}+\epsilon}(B C L M)^{\frac{1}{2}}}{N^{\frac{1}{2}}} .
$$

By the reasoning immediately after Proposition 5.2, the bound (7.2) allows us to assume $N \leq M Q^{-2 \epsilon}$, so that $N \leq Q^{\frac{1}{2}}$, regardless of whether $C L M \ll B$. In the case $C L M \ll B$, the bound (7.2) becomes

$$
\mathcal{E}_{2}^{\prime} \ll \frac{Q^{\frac{3}{2}+\epsilon} B}{N^{\frac{1}{2}}} \ll Q^{\frac{3}{2}+\epsilon} B \ll Q^{\frac{3}{2}+\theta_{2}+\epsilon} \ll Q^{2-\epsilon},
$$

which of course is acceptable.

At this point we can follow the rest of the proof in section 5. With $N \leq Q^{\frac{1}{2}}$ we can apply the reciprocity formula (5.7) and show the error term is sufficiently small. After changing variables $b n \rightarrow n$ the rest follows mutatis mutandis. This completes the proof of Lemma 3.4 .

\section{ACKNOWLEDGMents}

The author thanks Kevin Ford and George Shakan for helpful comments on earlier drafts of this work. The author was supported in this work by NSF grant DMS-1501982, and is grateful to Kevin Ford for financial support.

\section{REFERENCES}

[1] R. Balasubramanian, V. K. Murty. Zeros of Dirichlet L-functions. Ann. Sci. École Norm. Sup. (4) 25 (1992), no. 5, 567-615

[2] V. Blomer, É. Fouvry, E. Kowalski, P. Michel, D. Milićević. On moments of twisted L-functions. Amer. J. Math. 139 (2017), no. 3, 707-768 
[3] E. Bombieri, J. B. Friedlander, H. Iwaniec. Primes in arithmetic progressions to large moduli. Acta Math. 156 (1986), no. 3-4, 203-251

[4] H. M. Bui. Non-vanishing of Dirichlet L-functions at the central point. Int. J. Number Theory 8 (2012), no. $8,1855-1881$

[5] H. M. Bui, M. B. Milinovich. Central values of derivatives of Dirichlet L-functions. Int. J. Number Theory 7 (2011), no. 2, 371-388

[6] J. B. Conrey, H. Iwaniec, K. Soundararajan. Asymptotic large sieve. Preprint, May 2011, https://arxiv.org/abs/1105.1176

[7] J.-M. Deshouillers, H. Iwaniec. Kloosterman sums and Fourier coefficients of cusp forms. Invent. Math. $70(1982 / 83)$, no. 2, 219-288

[8] W. Duke, J. B. Friedlander, H. Iwaniec. Bilinear forms with Kloosterman fractions. Invent. Math. 128 (1997), no. 1, 23-43

[9] H. Iwaniec, E. Kowalski. Analytic number theory. American Mathematical Society Colloquium Publications, 53. American Mathematical Society, Providence, RI, 2004. xii+615 pp.

[10] H. Iwaniec, P. Sarnak. Dirichlet L-functions at the central point. Number theory in progress, Vol. 2 (Zakopane-Kościelisko, 1997), 941-952, de Gruyter, Berlin, 1999

[11] R. Khan, H. T. Ngô. Nonvanishing of Dirichlet L-functions. Algebra Number Theory 10 (2016), no. 10, 2081-2091

[12] P. Michel, J. VanderKam. Non-vanishing of high derivatives of Dirichlet L-functions at the central point. J. Number Theory 81 (2000), no. 1, 130-148

[13] S. J. Miller, R. Takloo-Bighash. An invitation to modern number theory. With a foreword by Peter Sarnak. Princeton University Press, Princeton, NJ, 2006.

[14] F. Sica. The order of vanishing of L-functions at the center of the critical strip. McGill University, $\mathrm{PhD}$ thesis, 1998

[15] K. Soundararajan. Mean-values of the Riemann zeta-function. Mathematika 42 (1995), no. 1, 158-174

[16] K. Soundararajan. Nonvanishing of quadratic Dirichlet L-functions at $s=\frac{1}{2}$. Ann. of Math. (2) 152 (2000), no. 2, 447-488

Department of Mathematics, University of Illinois, 1409 West Green Street, Urbana, IL 61801, United States

E-mail address: kpratt4@illinois.edu 